\title{
Vibration control by piezoelectric proof-mass absorber with resistive-inductive shunt
}

\author{
Høgsberg, Jan
}

Published in:

Mechanics of Advanced Materials and Structures

Link to article, DOI:

10.1080/15376494.2018.1551587

Publication date:

2021

Document Version

Peer reviewed version

Link back to DTU Orbit

Citation (APA):

Høgsberg, J. (2021). Vibration control by piezoelectric proof-mass absorber with resistive-inductive shunt. Mechanics of Advanced Materials and Structures, 28(2), 141-153 .

https://doi.org/10.1080/15376494.2018.1551587

\section{General rights}

Copyright and moral rights for the publications made accessible in the public portal are retained by the authors and/or other copyright owners and it is a condition of accessing publications that users recognise and abide by the legal requirements associated with these rights.

- Users may download and print one copy of any publication from the public portal for the purpose of private study or research.

- You may not further distribute the material or use it for any profit-making activity or commercial gain

- You may freely distribute the URL identifying the publication in the public portal

If you believe that this document breaches copyright please contact us providing details, and we will remove access to the work immediately and investigate your claim. 


\section{Vibration Control by Piezoelectric Proof-Mass Absorber WITH RESISTIVE-INDUCTIVE SHUNT}

\section{JAN HøGSBERG}

Department of Mechanical Engineering, Technical University of Denmark, Nils Koppels Allé, building 403, DK-2800 Kongens Lyngby, Denmark email: jhg@mek.dtu.dk, ORCID: 0000-0002-1098-3245.

\section{FUNDING}

This work was supported by the Independent Research Fund Denmark under Grant DFF-FP1 project 6111-00132A. 


\title{
Vibration Control by Piezoelectric Proof-Mass Absorber WITH RESISTIVE-INDUCTIVE SHUNT
}

\begin{abstract}
Vibration control of structures is introduced by a piezoelectric proof-mass absorber with a resistive-inductive shunt, introducing the structural mass, absorber mass and voltage across the shunt as the three degrees of freedom. The system poles follow semi-circular paths in the complex plane, whereby equal damping is secured by inverse point conditions for the undamped roots associated with vanishing and infinite resistance. The remaining parameters are calibrated relative to a reference solution with a triple-root bifurcation point. Design procedures are presented for parallel and series shunts, demonstrating a flat plateau in the amplitude curves for sufficiently separated root loci without bifurcation points.
\end{abstract}

\section{INTRODUCTION}

Proof-mass absorbers or tuned mass dampers are vibration absorbers that rely on an auxiliary absorber mass or inertia to create a supplemental resonance, which may improve the energy dissipation and thereby the overall performance when designed accurately with respect to a dominant vibration mode of the flexible structure. Alternative vibration absorption can be realized by piezoelectric transducers (laminate patches or stack actuators) with a supplemental resistive-inductive (RL) shunt to create the desired resonance [1, 2, 3, 4]. While mechanical absorbers with an auxiliary mass are effectively placed where the structural vibrations are large, the piezoelectric transducers act on relative displacement and thus commonly located where the deformations are largest. The calibration of shunt damping is conducted thoroughly by [4], specifying the electromechanical coupling coefficient and investigating the influence of structural damping on the calibration accuracy. A comprehensive summary of tuning expressions for both series and parallel RL shunts with respect to the minimization of displacement, velocity or acceleration amplitudes is provided in [5]. In [6] the inherent structural damping is included in the shunt resistive tuning, furthermore demonstrating that robustness improves with increased shunt damping. The validity of using a single mode assumption when analysing structures with piezoelectric vibration absorbers has recently been questioned in [7], based on results obtained for a flexible plate with a pair of shunted piezoelectric patches.

In the present paper the combined proof-mass absorber is considered, with an RL shunted piezoelectric transducer acting between the structure and the absorber mass. Piezoelectric proof-mass absorbers have originally been proposed for effective energy harvesting from ambient structural vibrations, where the apparent viscous component extracts the vibration energy created by the relative motion between structure and absorber mass. In the original concept $[8,9]$ a pure resistive $(\mathrm{R})$ shunt is implemented to extract the vibration energy based on an assumed harmonic motion of the host structure. Subsequently, the simple resistive shunt has been augmented by a supplemental inductance [10,11], creating the classic RL shunt well known from piezoelectric

vibration control [2]. However, as the motion of the host structure is commonly prescribed in a 
harvesting analysis, the optimization of the RL shunt components in the proof-mass absorber only involves a two degree-of-freedom (dof) system, whereby the solution procedure concerns a quartic characteristic equation. Therefore, the energy harvesting system analyzed in for example [11] is seemingly of the same order as for vibration control of structure by the classic tuned mass damper (TMD) $[12,13]$.

In vibration control and structural damping the dynamic or modal interaction between the flexible structure and the vibration absorber is essential, as damping and response mitigation requires that the motion of the structure is transferred and then converted into relative motion of the absorber mass by a proper synchronization of the two dofs in the combined system. Thus, in vibration control analysis the structural motion should appear as an independent system variable and may therefore not be prescribed, as otherwise assumed in most energy harvesting problems. The design and tuning of vibration or proof-mass absorbers is commonly founded on the classic results for the TMD $[12,13,14,15]$. As also demonstrated in the present paper, an RL shunted piezoelectric transducer acts equivalently to a spring-dashpot-inerter absorber, in which the dashpot/inerter component (representing the shunt) is connected in series with the equivalent spring (representing the capacitive property of the piezoelectric transducer). Thus, calibration principles and strategies may be obtained from the analysis of mechanical inerter-based TMDs, as reviewed in [15]. In [16] the viscous component of the TMD is replaced by a spring in series with a parallel dashpot-inerter element, for which a flat plateau in the frequency amplitude curve is obtained by a numerical optimization procedure. Furthermore, this preliminary analysis demonstrates that improved reduction in vibration amplitude is obtained for this inerter-based TMD compared to the classic TMD. The proposed absorber in [16] has recently been analyzed and numerically optimized in [17] with respect to random excitation acting either directly on the structure or as prescribed base motion. The inerter-based TMD involves three complex poles and therefore a substantially more complicated analysis than for the two-pole TMD system. However, closed-form solutions have actually been derived in [18] using a fixed-point approach similar to that used for the classic TMD in [12]. Although the derivations are quite extensive, simple expressions have indeed been obtained and validated against numerical optimization results. Passive inerter-based TMDs have also been applied to flexible structures with multiple dofs $[19,20]$ in which case the performance depends on the location, while the influence of non-resonant modes also interferes [21].

The application and/or tuning of piezoelectric proof-mass absorbers have been analyzed for structural vibration control. The closed-form solutions for an $H_{2}$-optimization have been presented in [22] for a series RL shunt. The analysis minimizes a measure for kinetic energy and results in fairly elongated expressions that are then substantially simplified by consistent truncations. An application of this RL shunted proof-mass absorber is then considered in [23], where structural vibrations in rotating machinery are targeted by the absorber system. An electromechanical proof-mass absorber has furthermore been considered for combined damping and energy harvesting in [24] using a pure resistive shunt without a shunt capacitance, thereby retaining an apparent two-dof system. 
In [25] a similar resistive shunt is used for energy harvesting in rotating machinery, similar to the problem considered in [23].

The performance of proof-mass absorbers may be further improved by active feedback control, which has been implemented in acceleration feedback in [26, 27], while a sky-hook principle is proposed in [28, 29] for a TMD with an additional inerter element and in [30] for a classic TMD with a spring-dashpot sky-hook element realized by non-collocated PD-control. Various virtual absorber systems are summarized and discussed in [31] with the aim of reducing/avoiding machinetool chatter vibrations.

The aim of the present paper is to tune RL shunts for vibration control by piezoelectric proofmass absorbers. It is initially demonstrated that the equivalent mechanical model corresponds to a classic TMD, where the viscous component is replaced by a spring in series with either a series or parallel dashpot-inerter element, corresponding to a parallel or series RL shunt, respectively. The shunt also contains a capacitor, comprising an $\mathrm{RL}(\mathrm{C})$ shunt, where the $C$ is stated inside parentheses, as it merely alters the inherent transducer capacitance. However, as demonstrated in $[32,33]$ the introduction of a negative capacitance component may improve the shunt performance significantly. The present dynamic system contains three dofs: The structure mass, the absorber mass and the resonance of the $\mathrm{RL}(\mathrm{C})$ shunt represented by the voltage across its terminals. Hereby, a pole-placement tuning procedure involves the handling of three complex poles. The present approach relies on the principle of equal modal damping, introduced in [13] for the TMD, subsequently generalized to resonant feedback equations in [34] and applied for the calibration of piezoelectric transducers with RL shunts in [35]. In the present case it is recognized that the individual root loci obtained with the shunt resistance as gain parameter describe half-circles in the complex plane. Thus, equal damping can be secured by choosing a mechanical stiffness ratio and a shunt mass ratio, representing the shunt inductance, such that the real-valued roots for a pure inductive shunt appear as inverse points with respect to a reference frequency, governed by short-circuited electrodes for the parallel shunt and open-circuited electrodes for the series RL shunt. Once the calibration formulae for these basic absorber parameters have been obtained, a reference solution with a triple-root bifurcation point is identified. A calibration procedure for a shunt stiffness ratio and shunt damping parameter, respectively representing capacitance and resistance, is derived relative to this unique reference solution. A simple parameter study finally demonstrates that a solution with respect to effective vibration mitigation is associated with two local root loci without any bifurcation point. This results in a fairly simple design procedure for the piezoelectric proof-mass absorber. The frequency response curves demonstrate that the present absorber outperforms the classic TMD, as also observed in [18] with cases c3 and c6 corresponding to the present problems with parallel and series RL shunts, respectively.

\section{THE PIEZOELECTRIC VIBRATION ABSORBER}

In the present problem a linear piezoelectric proof-mass absorber with absorber mass $m_{p}$ is applied to damp or mitigate vibrations of a linear host structure. Figure 1(a,c) shows the vibration 
problem with a piezoelectric (stack) transducer connecting the (modal) mass $m_{s}$ of the structure and the absorber mass $m_{p}$. The transducer is a solid-state device with mechanical stiffness $k_{p}$ and electromechanical coupling properties that can be used to modify the time dependent properties by a supplemental electrical circuit with impedance $Z$. Details concerning piezoelectric vibration control can be found in various textbooks $[2,3]$.

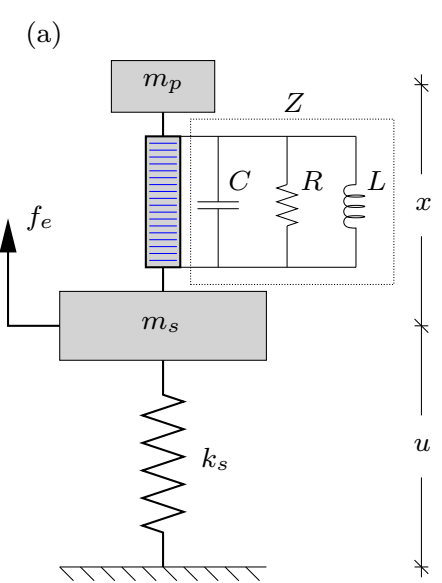

(c)

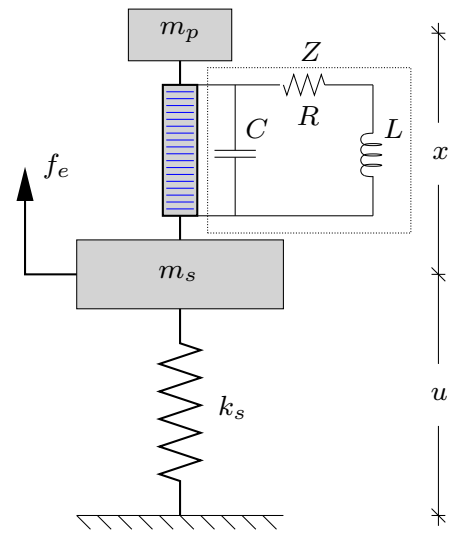

(b)

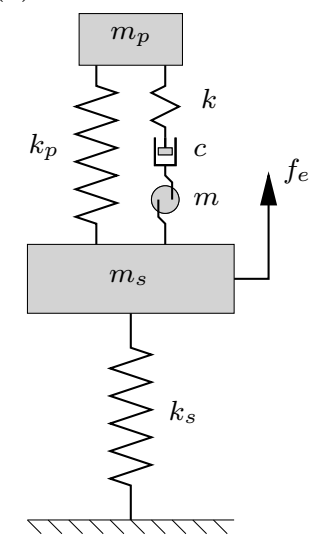

(d)

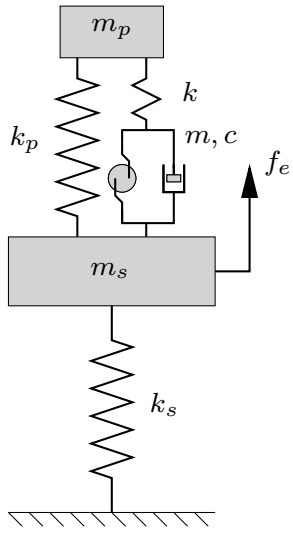

FiguRE 1. Single-mass vibrating structure $\left(m_{s}\right)$ with a shunted piezoelectric proof-mass absorber $\left(m_{p}\right)$. Parallel (a) and series (c) RL(C) shunts and equivalent mechanical model with series (b) and parallel (d) damper-inerter element, respectively.

2.1. Transducer force. The analysis is conducted in the frequency domain with time dependency represented by the exponential factor $\exp (i \omega t)$, containing the imaginary unit $i$, time $t$ and angular frequency $\omega$. In the following, the system variables therefore represent vibration amplitudes in the associated frequency response solution.

The supplemental transducer force from the shunt is denoted $f$, whereby the resulting transducer force is obtained by adding the contribution from the mechanical transducer stiffness. The resulting 
transducer force is therefore given as

$$
f_{p}=k_{p} x+f
$$

when $x$ represents the transducer deformation, as indicated in Fig. 1. For $x>0$ the structural mass $m_{s}$ experiences $f_{p}$ as an external force, whereby the governing equation for the structure may be written as

$$
\left(-\omega^{2} m_{s}+k_{s}\right) u-f_{p}=f_{e}
$$

Consequently, the absorber mass equation contains the transducer force with opposite sign,

$$
-\omega^{2} m_{p}(u+x)+f_{p}=0
$$

Hereby, (1) to (3) constitute the governing equations for the structure displacement $u$, transducer elongation $x$ and electromechanical force $f$.

The force relation (1) neglects any damping or hysteresis effects in the piezoelectric material, while intrinsic structural damping is furthermore omitted in (2). Although both effects may be nonnegligible and thus affect the calibration accuracy [6, 36], simple tuning expressions are however commonly derived for simplified models with damping only introduced by the dedicated shunt resistance $[5,4]$.

2.2. Electromechanical force. As already discussed, the conversion between electrical and mechanical energy is represented by the (effective) electromechanical coupling coefficient (EMCC) $\theta$, which determines the magnitude of the electromechanical force $f$ relative to the applied voltage $V$ across the transducer electrodes as

$$
f=\theta V
$$

The voltage is simultaneously governed by the sensor equation

$$
Q=-\theta x+C_{p} V
$$

where $Q$ is the charge stored at the transducer electrodes, while $C_{p}$ is the inherent capacitance associated with restrained deformation of the transducer $(x=0)$.

The specific electromechanical forcing is governed by the specific impedance of the applied shunt, conveniently defined by the relation $V=-i \omega Z(\omega) Q$ with $Z(\omega)$ representing the impedance function, see Fig. 1(a,c). Substitution of $Q$ in (5) into this impedance relation, followed by elimination of voltage by substitution into (4) gives the equivalent mechanical relation for the electromechanical force. This expression may be expressed by the flexibility relation

$$
x=\left(\frac{1}{C_{p}^{-1}}+\frac{1}{i \omega Z(\omega)}\right) \frac{f}{\theta^{2}}
$$

which corresponds to a spring with stiffness $\theta^{2} C_{p}^{-1}$ in series with a dashpot with frequency dependent viscous coefficient $\theta^{2} Z(\omega)$.

Dielectric losses in piezoelectric transducers and shunts may not always be negligible and commonly represented as supplemental (frequency dependent) model resistors [36, 37], which substantially complicate the derivation of analytical tuning expressions [5]. Thus, the tuning formulae presented 
in the following are only accurate for devices and components with sufficiently small dielectric losses compared to the overall level of modal damping introduced by the proof-mass absorber.

2.2.1. Parallel shunt. Figure 1(a) shows a parallel $\mathrm{RL}(\mathrm{C})$ shunt, in which the shunt capacitance $C$ alters the inherent transducer capacitance, whereas inductance $L$ and resistance $R$ govern the shunt resonance and introduce energy dissipation, respectively. Because the three electrical components are connected in a parallel configuration, the individual reciprocal impedances add up, whereby the resulting impedance function $Z(\omega)$ can be determined from

$$
\frac{1}{Z(\omega)}=\frac{i \omega}{C^{-1}}+\frac{1}{R}+\frac{1}{i \omega L}
$$

Upon substitution of $1 / Z(\omega)$ into (6), the mechanical flexibility relation can be written as

$$
x=\left(\frac{1}{k}+\frac{1}{i \omega c}-\frac{1}{\omega^{2} m}\right) f
$$

introducing the equivalent stiffness, damping and inertance (relative inertia) as

$$
k=\theta^{2}\left(C_{p}+C\right)^{-1} \quad, \quad c=\theta^{2} R \quad, \quad m=\theta^{2} L
$$

It is immediately seen that the shunt capacitance can be used to alter the inherent transducer capacitance, whereby the attainable damping can be increased by the means of an artificially generated negative capacitance, as described in greater detail in e.g. [32]. The mechanical relation (8) corresponds to a spring, dashpot and inerter placed in series, as shown in Fig. 1(b).

2.2.2. Series shunt. The alternative series $R L(C)$ shunt is shown in Fig. 1(c), with the shunt resistor and inductor placed in series in the circuit. For this series configuration the impedance function becomes

$$
\frac{1}{Z(\omega)}=\frac{i \omega}{C^{-1}}+\frac{1}{R+i \omega L}
$$

in which the resistance and inductance are added in the latter reciprocal term. Substitution into $V=-i \omega Z(\omega) Q$, followed by elimination of the charge $Q$ in the sensor equation (5), determines a relation between voltage $V$ and transducer deformation $x$. Thus, the equivalent mechanical flexibility relation for the electromechanically shunted piezoelectric transducer can be obtained directly by substitution into (4)

$$
x=\left(\frac{1}{k}+\frac{1}{i \omega c-\omega^{2} m}\right) f
$$

This relation is expressed in terms of the equivalent mechanical components already defined in (9). Thus, for the series $R L(C)$ shunt, the equivalent mechanical system contains the dashpot and inerter placed in parallel, as shown in Fig. 1(d).

2.3. Structural vibrations. An expression for the absorber force $f_{p}$ is determined by substitution of $f$ from either (8) or (11) into (1), while the relative absorber motion $x$ can subsequently be eliminated by (3). This procedure yields a resulting expression for the absorber force $f_{p}$ relative to the structural motion $u$, and upon substitution of this expression into the original structural 
equation of motion (2), an expression for the frequency response function is derived. For the parallel shunt with flexibility relation (8) the frequency response function becomes

$$
\frac{u k_{s}}{f_{e}}=\frac{\left\{\left(-r^{2} \mu_{p}+\kappa_{p}\right)\left[-r^{2} \mu+i r \frac{\mu \kappa}{\beta}+\kappa\right]-r^{2} \mu \kappa\right\}}{\left(-r^{2}\left(1+\mu_{p}\right)+1\right)\{\ldots\}-\left(r^{2} \mu_{p}\right)^{2}[\ldots]}
$$

while for the corresponding series shunt (11) it appears as

$$
\frac{u k_{s}}{f_{e}}=\frac{\left\{\left(-r^{2} \mu_{p}+\kappa_{p}\right)\left[-r^{2} \mu+i r \beta+\kappa\right]+\left(-r^{2} \mu+i r \beta\right) \kappa\right\}}{\left(-r^{2}\left(1+\mu_{p}\right)+1\right)\{\ldots\}-\left(r^{2} \mu_{p}\right)^{2}[\ldots]}
$$

The square brackets and curly braces with dots in the denominators refer to the specific expressions given in the corresponding numerators. Both frequency response functions are conveniently normalized and thereby expressed in terms of non-dimensional quantities. Initially, the frequency is normalized as

by the natural frequency

$$
r=\frac{\omega}{\omega_{s}}
$$

$$
\omega_{s}=\sqrt{\frac{k_{s}}{m_{s}}}
$$

of the structure without the proof-mass absorber. Subsequently, normalization by $k_{s}$ defines the mechanical and electrical stiffness ratios

$$
\kappa_{p}=\frac{k_{p}}{k_{s}} \quad, \quad \kappa=\frac{k}{k_{s}}
$$

whereby the normalization of the frequency directly introduces the corresponding mass ratios

$$
\mu_{p}=\frac{m_{p}}{m_{s}} \quad, \quad \mu=\frac{m}{m_{s}}
$$

The damper parameter $c$ is finally normalized by the geometric mean of $k_{s}$ and $m_{s}$,

$$
\beta=\frac{c}{\sqrt{k_{s} m_{s}}}
$$

whereby the damper ratio $\beta$ appears as a type of loss factor with respect to the dynamic properties of the host structure.

The static amplification factor of the frequency response functions in (12) and (13) is $u k_{s} / f_{e}=1$, while the amplitude $\left|u k_{s} / f_{e}\right|$ vanishes in the high-frequency limit due to the single sextic $\left(r^{6}\right)$ term in the denominator. Vibration absorbers are commonly used because of their ability to effectively reduce vibration amplitudes in an often sufficiently broad frequency range around the dominating resonance $r \simeq 1$. Thus, most calibration procedures rely on a minimization of the dynamic amplification factor $\left|u k_{s} / f_{e}\right|$. However, the present calibration procedure initially secures equal damping in the dynamic vibration modes of the system, which provides a robust design independent of the particular loading of the structure. This equal modal damping approach follows the analysis in [13] for the classic tuned mass damper (TMD) and in [35] for RL-shunted piezoelectric patches. The calibration procedure outlined in the following is therefore concerned with the proper placement of the complex system poles, governed by the sextic characteristic equation obtained from the denominator of the individual frequency response functions in (12) and (13). The frequency response function $u k_{s} / f_{e}$ for a structure with a TMD is recovered by 
the limit $\kappa \rightarrow \infty$ together with $\mu \rightarrow \infty$ or 0 in (12) or (13), respectively. The performance of the piezoelectric proof-mass absorber is compared to the classic TMD in Section 5.2.

\section{Equal DAmping Design}

The basic resonance properties of the system are determined by the roots of the characteristic equation and thus the singularities of the denominator in the frequency response functions in (12) and (13). Effective damping and response mitigation may be directly related to having the identical amount of damping in all vibratory modes, as an otherwise unbalanced modal damping would result in a less damped mode yielding an undesirable local peak in the corresponding response spectrum.

3.1. Characteristic equation. The present calibration procedure is therefore based on a pole placement strategy, in which the trajectories of the complex poles are investigated by analyzing the sextic characteristic equations with three complex roots. In a similar pole placement analysis of the TMD, the property of equal damping is simply secured by the two complex poles being inverse points with respect to a reference frequency representing the natural frequency of the system when the absorber mass is rigidly attached to the structure mass, see e.g. [13]. A reference frequency $\omega_{0}$ therefore defines a corresponding non-dimensional frequency ratio

$$
\xi=\frac{\omega}{\omega_{0}}=\frac{r}{\Omega_{0}}
$$

relative to the reference frequency ratio

$$
\Omega_{0}=\frac{\omega_{0}}{\omega_{s}}
$$

This reference frequency (ratio) plays an important role in the design, as the equal damping property is obtained when the dynamic roots are inverse points with respect to a backbone trajectory emerging from $\omega_{0}$. A thorough description of the concept of equal modal damping can be found in [13] for the TMD and in [34] for more generic resonant control formats.

In the following the viscous damper ratio $\beta$ is chosen as the gain variable of the root locus analysis, whereby the associated root loci will appear (approximately) as half circles in the complex plane. Based on this half-circle assumption, the desired location of the roots in the two limits without damping $(\beta \rightarrow 0$ and $\beta \rightarrow \infty$ ) can be used to secure equal modal damping for finite values of $\beta$ and thus a characteristic equation is conveniently separated into its odd-power polynomial terms, proportional to the representative damper ratio, and the remaining even-power terms and constant term.

3.1.1. Parallel shunt. For the parallel shunt the characteristic equation is obtained from the denominator in (12). When introducing the frequency ratio $\xi$ the characteristic equation can be 
written as

$$
\begin{array}{r}
-\xi^{6}+\frac{1}{\Omega_{0}^{2}}\left(1+\frac{\kappa}{\mu}+\left(1+\mu_{p}\right) \frac{\kappa+\kappa_{p}}{\mu_{p}}\right) \xi^{4} \\
-\frac{1}{\Omega_{0}^{4}}\left(\frac{\kappa}{\mu}\left(1+\left(1+\mu_{p}\right) \frac{\kappa_{p}}{\mu_{p}}\right)+\frac{\kappa+\kappa_{p}}{\mu_{p}}\right) \xi^{2}+\frac{1}{\Omega_{0}^{6}} \frac{\kappa}{\mu} \frac{\kappa_{p}}{\mu_{p}} \\
+i \xi \frac{1}{\Omega_{0}} \frac{\kappa}{\beta}\left[\xi^{4}-\frac{1}{\Omega_{0}^{2}}\left(1+\left(1+\mu_{p}\right) \frac{\kappa_{p}}{\mu_{p}}\right) \xi^{2}+\frac{1}{\Omega_{0}^{4}} \frac{\kappa_{p}}{\mu_{p}}\right]=0
\end{array}
$$

It is seen that the damper ratio $\beta$ appears by its reciprocal value as a factor on all odd-power terms. Thus, for $\beta \rightarrow 0$ the governing roots are determined by the quadratic equation inside the square bracket in (21). This limit $(c=0)$ implies that the equivalent dashpot in Fig. 1(b) transfers no force, whereby the two roots represent the vibration modes for the coupled $m_{s}-m_{p}$ dynamic system, with only stiffness $k_{p}$ connecting the two masses. In the opposite limit $\beta \rightarrow \infty$ the dashpot fully locks and instantaneously transfers the force, whereby the inertance $m$ is activated as the third dynamic degree of freedom. The roots of this system are determined by the cubic equation constituted by the even-power terms and constant term in (21).

3.1.2. Series shunt. For the series $\mathrm{RL}(\mathrm{C})$ shunt the slightly modified frequency response function is obtained from (13) and upon introduction of $\xi$ the corresponding characteristic equation can be written as

$$
\begin{array}{r}
-\xi^{6}+\frac{1}{\Omega_{0}^{2}}\left(1+\frac{\kappa}{\mu}+\left(1+\mu_{p}\right) \frac{\kappa+\kappa_{p}}{\mu_{p}}\right) \xi^{4}-\frac{1}{\Omega_{0}^{4}}\left(\frac{\kappa}{\mu}\left(1+\left(1+\mu_{p}\right) \frac{\kappa_{p}}{\mu_{p}}\right)+\frac{\kappa+\kappa_{p}}{\mu_{p}}\right) \xi^{2}+\frac{1}{\Omega_{0}^{6}} \frac{\kappa}{\mu} \frac{\kappa_{p}}{\mu_{p}} \\
+i \xi \frac{1}{\Omega_{0}} \frac{\beta}{\mu}\left[\xi^{4}-\frac{1}{\Omega_{0}^{2}}\left(1+\left(1+\mu_{p}\right) \frac{\kappa_{p}+\kappa}{\mu_{p}}\right) \xi^{2}+\frac{1}{\Omega_{0}^{4}} \frac{\kappa_{p}+\kappa}{\mu_{p}}\right]=0
\end{array}
$$

in which the odd-power terms are proportional to the damper ratio $\beta$. This implies that the two-root limit corresponds to infinite shunt damping $\beta \rightarrow \infty$, associated with locking the dashpot placed in parallel with the inerter in Fig. 1(d). This two-root limit is slightly different than for the parallel shunt, as the connecting stiffness is increased to $k_{p}+k$, associated with opencircuit transducer electrodes. The opposite three-root limit is reached for vanishing shunt damping $(\beta \rightarrow 0)$, in which case the dashpot in Fig. 1(d) vanishes. It is seen that the two mechanical models in Figs. 1(b) and (d) coincide in the limits when the dashpots are removed, thus having identical even-power polynomial terms in (21) and (22).

3.2. Inverse point relation. The present calibration strategy is developed upon the assumption that the complex roots of the system describe half-circular trajectories with respect to the viscous damper ratio $\beta$ as absorber gain. Possible root locus diagrams in the $\xi^{2}$-domain are sketched for the parallel $\mathrm{RL}(\mathrm{C})$ shunt in Fig. 2, where the two circle markers (o) on the horizontal axis represent the roots $\xi_{-1}^{2}$ and $\xi_{+1}^{2}$ obtained in the limit $\beta \rightarrow 0$ and thus governed by the odd-power terms in (21), while the three square markers $(\square)$ denote the three real-valued roots $\xi_{-2}^{2}$, $\xi_{0}^{2}$ and $\xi_{+2}^{2}$ for $\beta \rightarrow \infty$, associated with the even-power terms in (21). As $\beta$ appears by its reciprocal value in (22), the circles and squares will respectively depict the three- and two-root limits for the series $\mathrm{RL}(\mathrm{C})$. 

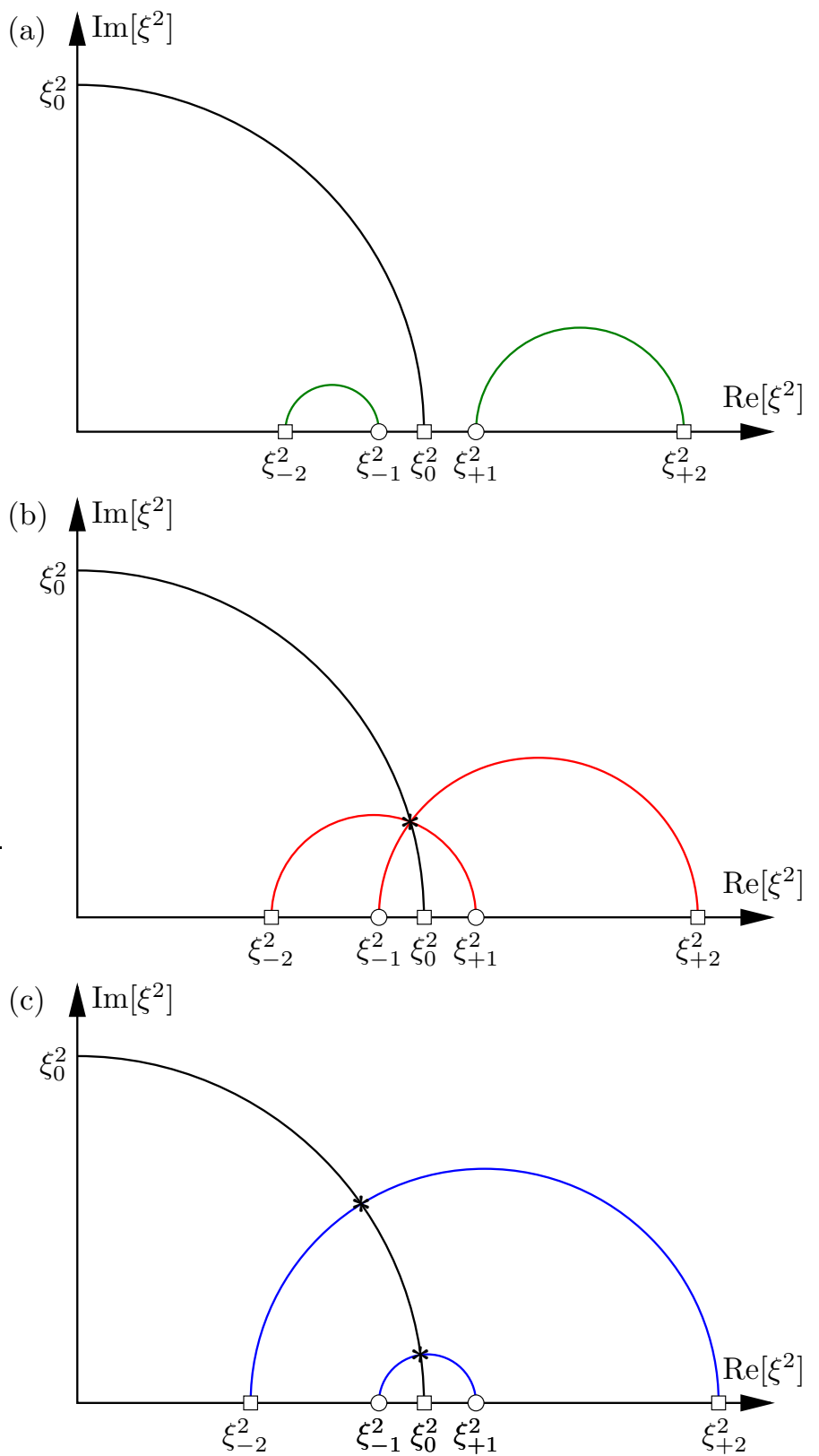

FiguRE 2. Generic root locus diagrams for structure with parallel RL(C) shunted piezoelectric proof mass absorber: (a) without bifurcation points, (b) with a single triple-root bifurcation point $(*)$ and $(c)$ with two double-root bifurcation points.

The initial aim of the absorber calibration is to secure that two of the three complex roots have equal damping. Following the approach in [13] for the TMD, this corresponds to the two complex roots being inverse points with respect to a unit quarter circle through the reference root $\xi_{0}^{2}=1$ on the horizontal axis. The third complex root must instead retain symmetry by following this unit backbone curve, represented in Fig. 2 by the black quarter circle. For a properly calibrated absorber system the third mode will have larger damping, whereby the attainable level of damping is governed by the two complex roots with equal damping. Based on the assumption of half-circular 
loci, the equal damping condition for the two complex poles can be realized by securing that each of the two pairs of real-valued roots $\xi_{\mp 1}^{2}$ and $\xi_{\mp 2}^{2}$ are themselves inverse points with respect to $\xi_{0}^{2}=1$ on the real axis. Hereby, a complicated design condition for fully complex roots is reduced to a simpler condition for the corresponding real-valued roots, determined by the characteristic equations obtained in the two limits $\beta \rightarrow 0$ and $\infty$.

The desired inverse point criteria on the real axis is determined by the conditions $\xi_{-1}^{2} \xi_{+1}^{2}=1$ in the two-root limit and correspondingly $\xi_{-2}^{2} \xi_{+2}^{2}=1$ in the three-root limit. For finite values of $\beta$ the trajectories of the vibratory complex roots will describe half circles in the complex plane, emerging and terminating at these real-valued roots. When the three-root poles $\xi_{\mp 2}^{2}$ are sufficiently close to the adjacent two-root poles $\xi_{\mp 1}^{2}$, the complex trajectory will pairwise connect the roots below and above the reference root $\xi_{0}^{2}=1$, as illustrated by the green half-circles in Fig. 2(a). If the three-root poles $\xi_{\mp 2}^{2}$ are instead sufficiently separated from their two-root counterparts $\xi_{\mp 1}^{2}$, a large locus must connect $\xi_{\mp 2}^{2}$, while a smaller locus between $\xi_{\text {干1 }}^{2}$ will determine the attainable damping in the system. These loci are shown as blue half-circular curves in Fig. 2(c). While the local green loci in (a) are without bifurcation points, the construction of the blue loci in (c) are associated with two bifurcation points (asterisks) on the black quarter circle. The intermediate case in Fig. 2(b) is therefore associated with a single bifurcation point on the black quarter circle, at which three roots meet simultaneously for a properly calibrated absorber with equal damping.

3.2.1. Parallel shunt. For the parallel $\mathrm{RL}(\mathrm{C})$ shunt the normalized characteristic equation is given in (21) with the three-root limit (squares) obtained by $\beta \rightarrow \infty$, while the corresponding two-root case (circles) is reached for vanishing $\beta$.

The condition that the real-valued roots $\xi_{\mp 1}^{2}$ for $\beta \rightarrow 0$ appear as inverse points with respect to $\xi_{0}^{2}=1$ requires a unit ratio between the coefficients to the first- and fifth-order terms in (21). This determines the first calibration condition

$$
\frac{\kappa_{p}}{\mu_{p}}=\Omega_{0}^{4}
$$

Similarly, a unit ratio between the coefficients to the sixth-order term and constant term is required to secure the inverse point relation for $\xi_{\text {干 } 2}^{2}$ in the limit $\beta \rightarrow \infty$,

$$
\frac{\kappa}{\mu} \frac{\kappa_{p}}{\mu_{p}}=\Omega_{0}^{6}
$$

A third condition is needed to normalize the reference root $\xi_{0}^{2}=1$. This is secured by having identical coefficients to the fourth- and second-order terms in (21). This condition can be formulated as

$$
\Omega_{0}^{2}\left[1+\frac{\kappa}{\mu}+\left(1+\mu_{p}\right) \frac{\kappa+\kappa_{p}}{\mu_{p}}\right]=\left[\frac{\kappa}{\mu}\left(1+\left(1+\mu_{p}\right) \frac{\kappa_{p}}{\mu_{p}}\right)+\frac{\kappa+\kappa_{p}}{\mu_{p}}\right]
$$

When (23) and (24) are substituted into (25) a solution for $\kappa$ can be obtained, whereby the three calibration expressions

$$
\kappa_{p}=\frac{\mu_{p}}{\left(1+\mu_{p}\right)^{2}} \quad, \quad \kappa=\frac{\mu}{1+\mu_{p}} \quad, \quad \Omega_{0}^{2}=\frac{1}{1+\mu_{p}}
$$


secure the desired inverse point property with respect to $\xi_{0}^{2}=1$. It follows from these relations that the stiffness ratios and the reference frequency are smaller than the corresponding mass ratios and unity, respectively. Furthermore, the mass ratio $\mu_{p}$ associated with the physical absorber mass appears to be a suitable absorber parameter, leaving the transducer stiffness ratio $\kappa$ and the shunt damper ratio $\beta$ as the remaining free calibration parameters to be determined.

3.2.2. Series shunt. In case of a series $\mathrm{RL}(\mathrm{C})$ shunt the characteristic equation is given as in (22) from which the three- (circles) and two-root (squares) solutions are obtained in the limits $\beta \rightarrow 0$ and $\infty$, respectively. As for the parallel shunt, the fifth-to-first and sixth-to-zero order coefficient ratios must be unity, while the coefficients to the fourth and second order polynomial terms must be identical. However, as the even-power terms are identical in the two characteristic equations (21) and (22), it is only the former of the three conditions that changes in the present case. Thus, (23) is simply replaced by

$$
\frac{\kappa_{p}+\kappa}{\mu_{p}}=\Omega_{0}^{4}
$$

while the remaining two conditions are identical to those in (24) and (25) found for the parallel shunt configuration. The resulting calibration expressions are then conveniently formulated as

$$
\kappa+\kappa_{p}=\mu_{p} \quad, \quad \kappa \kappa_{p}=\mu \mu_{p} \quad, \quad \Omega_{0}^{2}=1
$$

implying that the reference frequency $\omega_{0}$ is not shifted relative to the resonance frequency $\omega_{s}$.

\section{Complex Root Design}

The expressions in (26) and (28) secure $\xi_{0}^{2}=1$ and the desired inverse point relations $\xi_{-1}^{2} \xi_{+1}^{2}=1$ and $\xi_{-2}^{2} \xi_{+2}^{2}=1$ with respect to unity. However, as described in connection with Fig. 2 the particular type and shape of the overall root locus diagram depends on the relative distance between $\xi_{\mp 1}^{2}$ and $\xi_{\mp 2}^{2}$ on the real (horizontal) axis. If the roots from opposite $\beta$ limits are too far away, a large and a small (blue) half-circular trajectory will combine $\xi_{\mp 1}^{2}$ and $\xi_{\mp 2}^{2}$, respectively, while when they are sufficiently close local (green) trajectories will instead connect $\xi_{-2}^{2}$ to $\xi_{-1}^{2}$ and $\xi_{+1}^{2}$ to $\xi_{+2}^{2}$. In this section the condition for the intermediate case is determine, in which the three complex roots meet at a single bifurcation point on the unit quarter-circle. This intermediate case is described by the red half-circles in Fig. 2(b) with a triple-root bifurcation point (asterisk).

4.1. Triple-root bifurcation point. The characteristic equations for the parallel and series $\mathrm{RL}(\mathrm{C})$ shunt configurations are given in (21) and (22), respectively. Upon elimination of $\mu$ and $\Omega_{0}$ the following common equation can be obtained,

$$
-\xi^{6}+\left[3+\mu_{p}+\frac{\kappa}{\kappa_{p}}\right]\left(\xi^{4}-\xi^{2}\right)+1+i \xi \eta\left[\xi^{4}-\left(2+\mu_{p}\right) \xi^{2}+1\right]=0
$$

with the common damper gain

$$
\eta= \begin{cases}\frac{\mu \Omega_{0}}{\beta} & \text { parallel shunt } \\ \frac{\beta}{\mu \Omega_{0}} & \text { series shunt }\end{cases}
$$


representing reciprocal values for the two shunt configurations.

The existence of a triple-root bifurcation point, with complex conjugate frequency solutions, implies

$$
\left(\xi-\xi_{*}\right)^{3}\left(\xi-\bar{\xi}_{*}\right)^{3}=0
$$

where the bar represents the conjugate solution. The complex root at the bifurcation point then may be expressed as $\xi_{*}=a+i b$, in terms of its real part $a$ and imaginary part $b$. The conjugate solution is given as $\bar{\xi}_{*}=-a+i b$, whereby $\xi_{*}+\bar{\xi}_{*}=2 i b, \xi_{*}-\bar{\xi}_{*}=2 a$ and $\xi_{*} \bar{\xi}_{*}=-\left(a^{2}+b^{2}\right)=-\left|\xi_{*}\right|^{2}$. In terms of $a$ and $b$ the bifurcation condition (31) can be written as

$$
\xi^{6}+3\left(4 b^{2}+1\right)\left(\xi^{4}-\xi^{2}\right)+1+i \xi(6 b)\left\{\xi^{4}-2\left(\frac{2}{3} b^{2}+1\right) \xi^{2}+1\right\}=0
$$

where $\left|\xi_{*}\right|=1$ has been implied, as the bifurcation point lies on the assumed unit quarter circle. Comparison of the terms in (29) and (32) gives the solution to the bifurcation point

$$
\xi_{*}=\sqrt{1-\frac{3}{4} \mu_{p}}+i \sqrt{\frac{3}{4} \mu_{p}}
$$

and thereby

$$
\xi_{*}^{2}=1-\frac{3}{2} \mu_{p}+2 i \sqrt{\frac{3}{4} \mu_{p}\left(1-\frac{3}{4} \mu_{p}\right)}
$$

in the complex $\xi^{2}$-plane. Furthermore, it provides the stiffness ratio

$$
\frac{\kappa_{*}}{\kappa_{p}}=8 \mu_{p}
$$

and generic damper gain

$$
\eta_{*}=\sqrt{27 \mu_{p}}
$$

where the asterisk in the following refers to the reference solution with a single triple-root bifurcation point, as illustrated in Fig. 2(b). The solutions in (33) and (34) verify the assumed relation $\xi_{*} \bar{\xi}_{*}=-1$ and thus $\xi_{*}^{2} \bar{\xi}_{*}^{2}=1$. Furthermore, the bifurcation point is associated with the damper ratio $\beta_{*}=\mu \Omega_{0} / \sqrt{27 \mu_{p}}$ for the parallel shunt and $\beta_{*}=\mu \Omega_{0} \sqrt{27 \mu_{p}}$ for the corresponding series shunt.

4.2. Damper design. Although the (asterisk) calibration in (35) and (36) provides the largest attainable damping ratio, it is non-optimal with respect to minimization of response amplitudes, as undesirable modal interference occurs because three vibration forms in this case operate with exactly the same frequency. Thus, slightly less absorber damping may instead be optimal, as well known for a TMD [12, 13] and piezoelectric shunt damping [1, 35, 38]. Thus, two scaling parameters $\alpha$ and $\nu$ are now assigned as

$$
\kappa=\alpha \kappa_{*} \quad, \quad \eta=\nu \eta_{*}
$$

to investigate the optimal values of $\kappa$ and $\eta$ relative to the reference solutions in (35) and (36) for the triple-root bifurcation point.

The proposed calibration procedure is summarized in Table 1 for both the parallel and the series $\mathrm{RL}(\mathrm{C})$ shunt configuration. The absorber mass ratio $\mu_{p}$ is in both cases chosen as the absorber parameter, whereby the inverse point relation defines the reference frequency ratio $\Omega_{0}$. A design variable $\alpha=\kappa / \kappa_{*}$ defines the transducer stiffness ratio $\kappa$ relative to the reference value $\kappa_{*}=8 \mu_{p} \kappa_{p}$ 

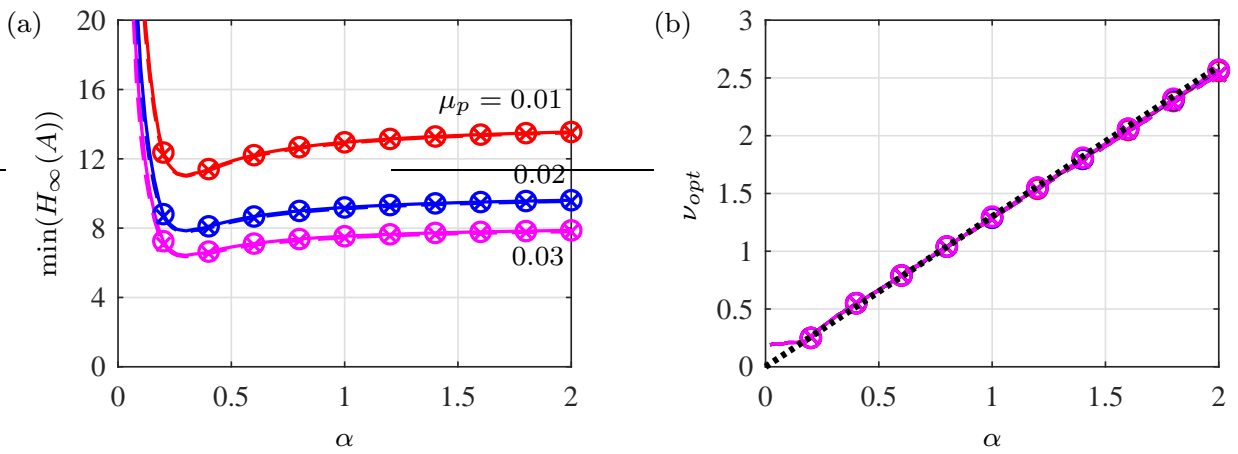

Figure 3. (a) Minimum of maximum vibration amplitude $A$ for $\mu_{p}=0.01$ (red), 0.02 (blue) and 0.03 (magenta). (b) Optimal damper factor $\nu_{\text {opt }}$. Shunt configuration: Parallel (o solid) and series ( $\times$ dashed). In (b) black dotted: $\nu_{o p t}=1.3 \alpha$.

defined in (35). Thus, $\alpha=1$ corresponds to the triple-root bifurcation point associated with the (red) half-circles in Fig. 2(b). Once $\alpha$ has been chosen the mechanical stiffness ratio $\kappa_{p}$ and transducer stiffness ratio $\kappa$ can be determined, whereafter the shunt inertance ratio $\mu$ may finally be found. The procedure is completed by choosing $\nu$ as the value of $\eta$ relative to the reference solution $\eta_{*}=\sqrt{27 \mu_{p}}$ in (36).

A numerical optimization analysis is initially performed to investigate the damping performance with respect to $\alpha$ and $\nu$. For a given value of $\mu_{p}$ and $\alpha$ the optimal value of $\nu$ is chosen so that the maximum vibration amplitude $A$ is minimized. For the parallel shunt the vibration amplitude $A$ is simply chosen as the dynamic amplification factor or compliance $A=\left|u k_{s} / f_{e}\right|$ [5], whereas the series shunt is effective with respect to the corresponding velocity amplitude or mobility $A=r\left|u k_{s} / f_{e}\right|$. The optimization is therefore equivalent to minimizing the $H_{\infty}$-norm of the particular vibration amplitude. Figure 3(a) shows the smallest maximum vibration amplitude $A$ as function of $\alpha$ for typical mass ratios $\mu_{p}=0.01$ (magenta), 0.02 (blue) and 0.03 (red), while Fig. 3(b) shows the corresponding optimal damper factor $\nu_{\text {opt }}$. The solid curves with circle markers

TABLE 1. Calibration procedure for given $\mu_{p}, \alpha=\kappa / \kappa_{*}$ and $\nu=\eta / \eta_{*}$.

\begin{tabular}{ccc}
\hline & Parallel & Series \\
\hline$\Omega_{0}^{2}$ & $\frac{1}{1+\mu_{p}}$ & 1 \\
$\kappa_{p}$ & $\frac{\mu_{p}}{\left(1+\mu_{p}\right)^{2}}$ & $\frac{\mu_{p}}{1+\alpha 8 \mu_{p}}$ \\
$\kappa$ & \multicolumn{2}{c}{$\alpha 8 \mu_{p} \kappa_{p}$} \\
$\mu$ & \multicolumn{2}{c}{$\kappa \frac{\kappa_{p}}{\mu_{p} \Omega_{0}^{6}}$} \\
$\frac{1}{\mu \Omega_{0}}$ & $\frac{1}{\nu \sqrt{27 \mu_{p}}} \quad \nu \sqrt{27 \mu_{p}}$ \\
\hline
\end{tabular}


(o) represent the results for the parallel $\mathrm{RL}(\mathrm{C})$ shunt, while the crosses $(x)$ represent the series shunt. It is seen that the two shunts are equally effective in reducing the respective vibration amplitudes and the optimal value of $\nu$ is seemingly proportional to $\alpha$. The black dotted line in Fig. 3(b) represents the linear relation $\nu_{o p t}=1.3 \alpha$, obtained by a linear regression for both shunts in the interval $0.5 \leq \alpha \leq 2$. Furthermore, it is seen from Fig. 3(a) that above a certain threshold around $\alpha=0.25$ the vibration amplitude increases with $\alpha$. An appropriate stiffness factor may therefore be $\alpha=0.5$ with $\nu=1.3 \alpha=0.65$ and a certain margin to the steep amplitude increase observed in Fig. 3(a) for $\alpha<0.25$.

\section{DyNAmic ANALYSis}

In the present section various results are presented for the single degree-of-freedom structure with a shunted piezoelectric proof-mass absorber. The coupled time domain equations of motion can be written in matrix form as

$$
\left(-\omega^{2} \mathbf{M}+i \omega \mathbf{C}+\mathbf{K}\right) \mathbf{q}=\mathbf{f}
$$

where the augmented displacement (column) vector

$$
\mathbf{q}=\left[u, x, f / k_{s}\right]^{T}
$$

contains the structure and absorber mass displacements and the normalized electromechanical force. For both shunt configurations the mass and stiffness matrices are

$$
\mathbf{M}=\left[\begin{array}{rrr}
1 & 0 & 0 \\
\mu_{p} & \mu_{p} & 0 \\
0 & -\mu & \mu
\end{array}\right], \quad \mathbf{K}=\omega_{s}^{2}\left[\begin{array}{rrr}
1 & -\kappa_{p} & -\kappa \\
0 & \kappa_{p} & \kappa \\
0 & 0 & \kappa
\end{array}\right]
$$

while the damping matrix is given as

$$
\mathbf{C}_{\mathrm{par}}=\omega_{s}\left[\begin{array}{ccc}
0 & 0 & 0 \\
0 & 0 & 0 \\
0 & 0 & \mu \kappa / \beta
\end{array}\right], \quad \mathbf{C}_{\mathrm{ser}}=\omega_{s}\left[\begin{array}{ccc}
0 & 0 & 0 \\
0 & 0 & 0 \\
0 & -\beta & \beta
\end{array}\right]
$$

for the parallel and series $\mathrm{RL}(\mathrm{C})$ shunt, respectively.

5.1. Root locus analysis. The root locus analysis is conducted by tracing the system poles in the complex plane for increasing damper ratio $\beta$. The complex poles are determined by solving the eigenvalue problem associated with $\mathbf{f}=\mathbf{0}$ in (38). The poles could also be obtained as the roots to the characteristic equations in (21) and (22).

Figure 4 shows the root loci for both the parallel $\mathrm{RL}(\mathrm{C})$ shunt $(\mathrm{a}, \mathrm{c}, \mathrm{e})$ and series shunt $(\mathrm{b}, \mathrm{d}, \mathrm{f})$ with absorber mass ratio $\mu_{p}=0.02$, while $\alpha=0.5(\mathrm{a}, \mathrm{b}), 1.0(\mathrm{c}, \mathrm{d})$ and 2.0 (e,f). The real-valued limits $\beta \rightarrow 0$ and $\infty$ are indicated by $\circ$ and $\square$, respectively. Thus, the squares denote the three-root limit for the parallel shunt and consequently the two-root limit for series shunt, and vice versa for the circles. The red dots represent the desired calibration $\nu=1.3 \alpha$, while the asterisks instead refer to the solution $\nu=\alpha$ that recovers the bifurcation point $(\nu=1)$ for $\alpha=1$. It is seen in Fig. $4(\mathrm{c}, \mathrm{d})$ that the asterisks actually coincide exactly at the bifurcation point for both shunt configuration. However, in the optimal solution the two vibratory poles have identical damping at solutions below the bifurcation point, while the third pole is then more damped above the bifurcation point on 

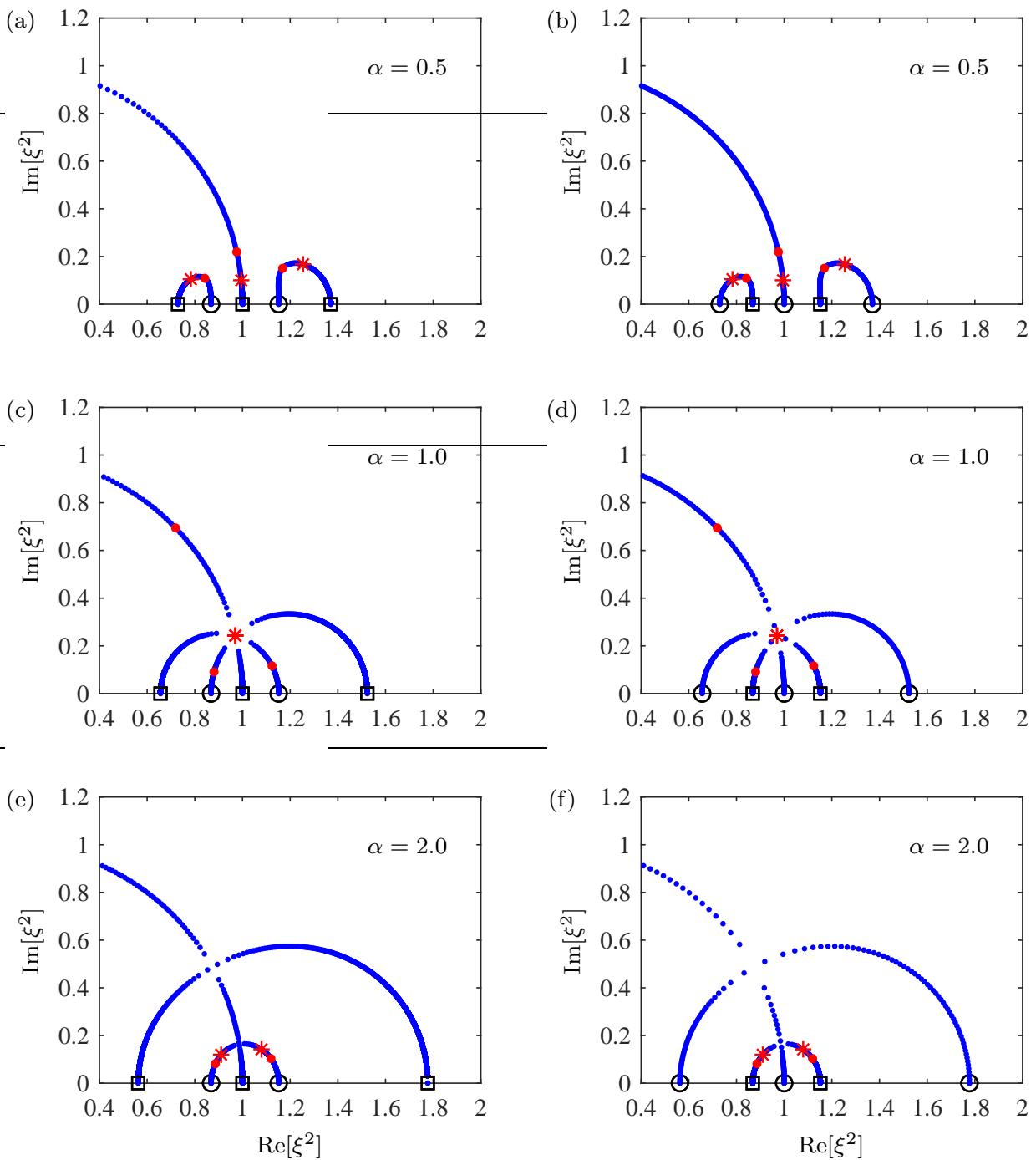

FiguRE 4. Root locus diagrams for parallel (a,c,e) and series (b,d,f) )shunt with $\mu_{p}=0.02$ and $\alpha=0.5(\mathrm{a}, \mathrm{b}), 1.0(\mathrm{c}, \mathrm{d})$ and $2.0(\mathrm{e}, \mathrm{f})$. Damper factor $\nu=1.3 \alpha(\bullet)$ and $\nu=1.0 \alpha(*)$. Real valued limits $\beta \rightarrow 0$ by $\circ$ and $\infty$ by $\square$.

the unit quarter circle. For $\alpha=2.0$ the third pole in Fig. $4(\mathrm{e}, \mathrm{f})$ is not even within the chosen figure boundaries, while the vibratory poles are located on the inner small half-circle, basically recovering the TMD solution in [13]. For the preferred calibration with $\alpha=0.5$ in Fig. 4(a,b) the vibratory poles are placed at the top of the two local loci, while the third pole is only slightly more damped. This $(\alpha=0.5)$ design seems therefore to have a more pronounced interaction between the three modes, while still avoiding the creation of a bifurcation point. An inherent problem with bifurcation points is furthermore that the location of the poles is very sensitive with respect to rather small changes in the gain variable. The blue dots, describing the loci, are obtained for equidistant value of $\beta$ and around the triple-root bifurcation point in Fig. 4(c,d) the rate of change is visibly very large. The complex roots and corresponding damping ratios $\zeta=\operatorname{Im}[\xi] /|\xi|$ are summarized in Table 2 for both the poles associated with $\nu=1.3 \alpha(\bullet)$ and $\nu=1.0 \alpha(*)$. 
TABLE 2. Damping ratio $\zeta$ for the three modes of the system with a parallel $\mathrm{RL}(\mathrm{C})$ shunt: $\mu_{p}=0.02$ and $\nu=1.3 \alpha$ or $1.0 \alpha$.

\begin{tabular}{cccc}
\hline$\nu / \alpha$ & $\alpha=0.5$ & $\alpha=1.0$ & $\alpha=2.0$ \\
\hline $1.3(\bullet)$ & $0.064,0.110,0.064$ & $0.052,0.375,0.052$ & $0.046,0.863,0.046$ \\
$1.0(*)$ & $0.066,0.050,0.066$ & $0.123,0.123,0.123$ & $0.065,0.604,0.065$ \\
\hline
\end{tabular}
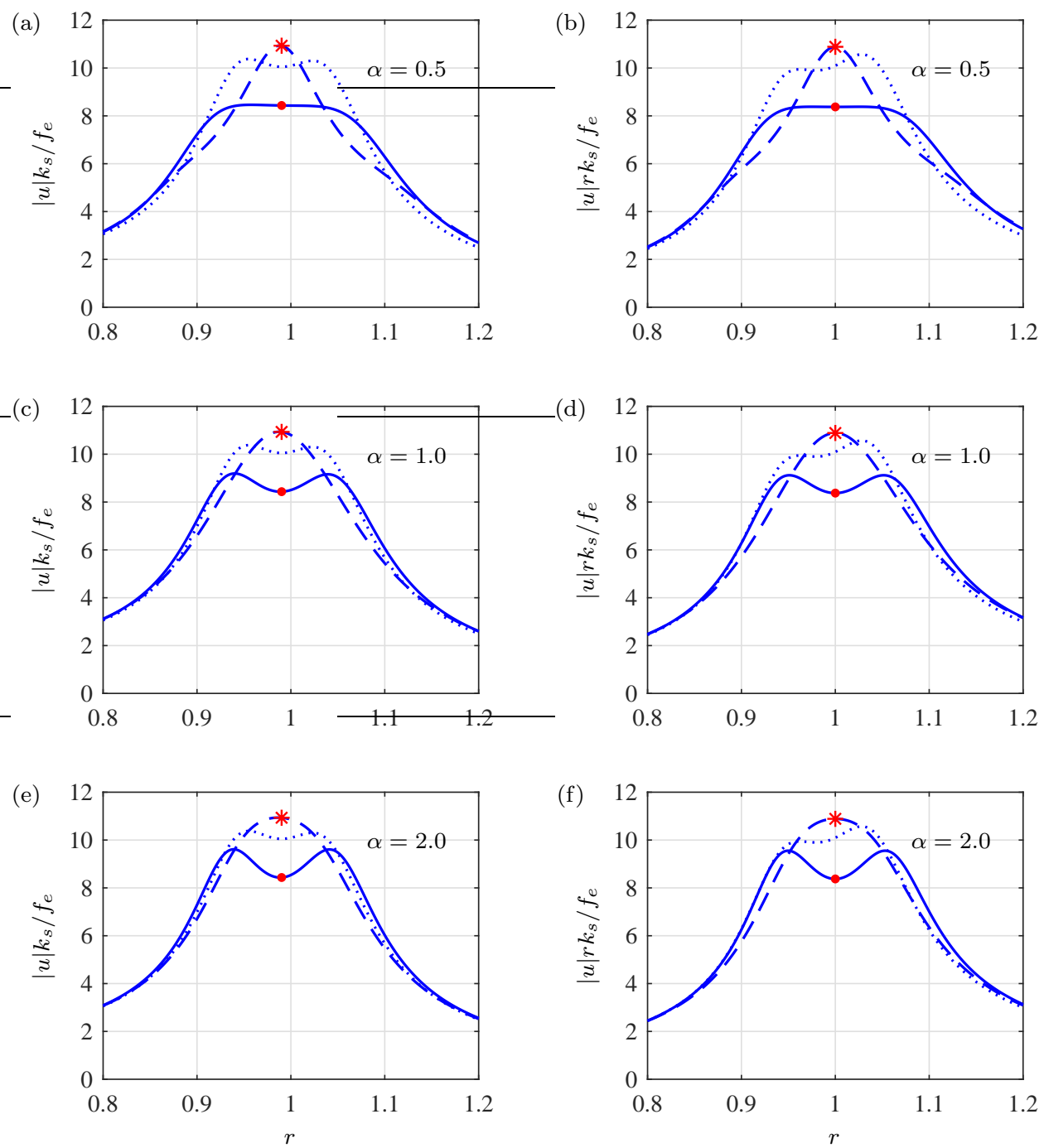

FIGURE 5. Vibration amplitude curves for parallel (a,c,e) and series (b,d,f) shunt with $\mu_{p}=0.02$ and $\alpha=0.5(\mathrm{a}, \mathrm{b}), 1.0(\mathrm{c}, \mathrm{d})$ and $2.0(\mathrm{e}, \mathrm{f})$. Damper factor $\nu=1.3 \alpha$ (solid $\bullet$ ) and $\nu=1.0 \alpha$ (dashed $*$ ). Mechanical tuned mass damper [13] (dotted).

For the proposed case $(1.3 \alpha)$ the redundant mode is always more damped than the two vibratory modes with equal damping ratios, while for $\nu=1.0 \alpha$ and $\alpha=0.5$ the attainable damping is actually limited by the redundant intermediate mode. The damping ratios are slightly larger for $\alpha=0.5$ than for $\alpha=1.0$ and 2.0, indicating that for $\alpha$-values in this range the absorber combines large damping with effective response mitigation. 
(a)

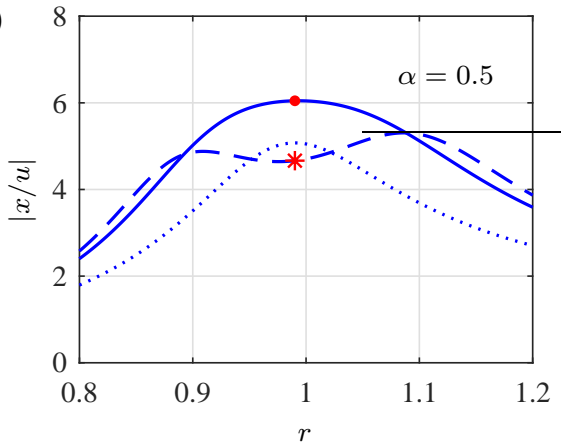

(b)

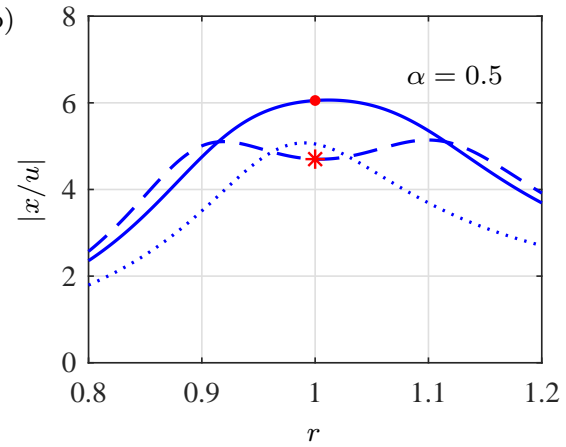

FiguRE 6. Deformation of piezoelectric transducer $x$ relative to structural amplitude $u$ for parallel (a) and series (b) shunt with $\mu_{p}=0.02$ and $\alpha=0.5$. Damper factor $\nu=1.3 \alpha($ solid $\bullet)$ and $\nu=1.0 \alpha($ dashed $*)$. Mechanical tuned mass damper [13] (dotted).

5.2. Frequency response analysis. The vibration amplitudes are investigated by load vector $\mathbf{f}^{T}=\left[f_{e} / m_{s}, 0,0\right]$ without external loading on the absorber mass and transducer. The system of equations (38) is solved for various angular frequencies $\omega$ around the structure resonance $\omega_{s}$ and Fig. 5 shows the displacement amplitude $\left|u k_{s} / f_{e}\right|$ for the parallel shunt (a,c,e) and the velocity amplitude $\left|u r k_{s} / f_{e}\right|$ for the series shunt (b,d,f). The desired flat plateau in the amplitude curve around the resonance is obtained for both shunts, clearly indicating that the parallel shunt should be chosen when aiming at reducing the displacement, whereas in cases where the velocity is critical the series shunt instead provides the desired behavior. For $\alpha=0.5$ and $\nu=1.3 \alpha=0.65$ the amplitude is practically flat, demonstrating that the absorber calibration is well-balanced without any local peaks. For increasing $\alpha$ two significant peaks appear with a pronounced trough in between. And for $\alpha=1.0$ and 2.0 the shape of the amplification curves correspond very much to that of a classic TMD [13], represented by the dotted curves in Fig. 5. Comparison of the solid and dotted curves furthermore indicate the additional reduction in vibration amplitude realized by the LC resonance of the shunt. In all cases the alternative tuning $\nu=1.0 \alpha$ yields a single peak (dashed) curve with a substantial increase in vibration amplitude. Thus, it is appropriate to choose a shunt resistance that gives a solution that is slightly below the bifurcation point. It follows from Table 2 that the proposed calibration provides damping ratios $(\zeta=0.06)$ that are approximately half of the damping ratio obtained at the bifurcation point $(\zeta=0.12)$.

A main drawback of vibration mitigation by vibration proof-mass absorbers is the inherent amplification of the absorber mass motion and thereby the deformation $x$ of the piezoelectric transducer. Figure 6 shows the transducer deformation $x$ relative to the structural amplitude $u$, which indicates that for $\mu_{p}=0.02$ the required transducer deformation is six times larger than the structural vibration level. Furthermore, the proposed calibration with $\nu=0.65$ (solid curves) results in larger deformations than for $\nu=0.5$ (dashed) and the equivalent results obtained for the classic TMD (dotted). Piezoelectric ceramics are solid state devices with brittle material properties and low fracture strain compared to the allowable deformation of the mechanical springs used in TMDs. 

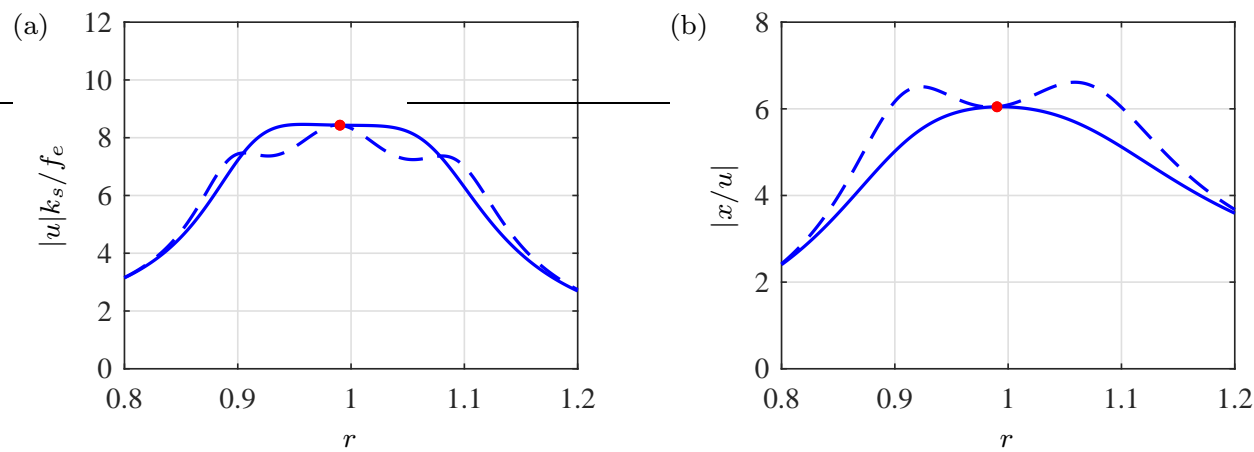

Figure 7. Amplitude curves for structural motion (a) and relative transducer deformation (b). Parallel shunt with $\mu_{p}=0.02$ and $\alpha=0.3$ (dashed) and 0.5 (solid).

Therefore, the reported deformation amplitudes in Fig. 6 may be a limiting factor for the applicability of piezoelectric proof-mass absorbers. At least this preliminary analysis suggests the use of mechanically amplified devices, in which the actual deformation of the transducer is substantially reduced relative to $x$ by a mechanical scaling.

A detailed analysis of the minimization curves in Fig. 3 reveal that the optimal stiffness ratio is obtained for $\alpha=0.3$. Figure 7 compares the frequency response amplitudes for this apparent optimum (dashed) with the proposed calibration value $\alpha=0.5$ (solid). The results are shown for the parallel shunt and therefore the two solid curves in Fig. 7 correspond to those in Fig. 5(a) and Fig. 6(a), respectively. The vibration amplitudes for $\alpha=0.3$ and 0.5 are identical at the reference frequency ratio $r=\Omega_{0}$ (red $\bullet$ ), while the small inclination of the solid curve implies that the vibration amplitude for $\alpha=0.5$ is in fact slightly larger than for $\alpha=0.3$ when $r<\Omega_{0}$. However, for $\alpha=0.3$ the relative deformation amplitude in Fig. 7(b) exhibits two pronounced peaks associated with amplified straining of the piezoelectric transducer. And since $\alpha=0.5$ furthermore maintains a desirable margin to $\alpha<0.25$, associated with an increase in vibration amplitude, this value appears to be appropriate.

\section{Conclusions}

Proof-mass absorbers are effectively applied because they optimally operate where the structure experiences the largest deflections. For piezoelectric proof-mass absorbers the performance can be adjusted by introducing a resistive-inductive (RL) shunt between the transducer electrodes, whereby the three governing degrees of freedom (dofs) of the dynamic system are comprised of the structural (modal) mass, the physical absorber mass and the supplemental shunt voltage. It is worth mentioning that for inductive shunts the third supplemental dof may not be eliminated because of the inherent absorber stiffness from the transducer capacitance.

A combined pole-placement and response minimization procedure is proposed for the design and tuning of the shunted absorber. Initially the robust principle of equal damping is secured for the complex roots. This determines expressions for the mechanical stiffness $\left(\kappa_{p}\right)$ of the transducer and 
the shunt inductance $(\mu)$. The remaining absorber parameters, representing the inherent transducer capacitance $(\kappa)$ and the shunt resistance $(\beta)$, are subsequently calibrated to minimize the frequency response amplitude. A reference root locus diagram is identified, with a single bifurcation point for three complex roots. However, it is well known that bifurcation points introduce constructive response interference and thus increased vibration amplitudes. A simple numerical search procedure is therefore used to identify principles associated with minimum vibration amplitude. The analysis shows that a calibration without any bifurcation points results in improved absorber performance, combining both effective response mitigation and large (modal) damping. The optimal condition is seemingly characterized by a sufficient interaction between the three complex modes, without the roots meeting at a bifurcation point. A simple tuning procedure is presented for both the parallel and the series shunt configurations. A dynamic analysis in Fig. 5 demonstrates an improved performance over the classic TMD, as also reported in $[16,17]$ for vibration absorbers augmented by inerters. However, as the equivalent inerter elements in Fig. 1 are realized electronically by transducer shunts, the performance and calibration accuracy do not rely on assumptions of weightless inerter elements.

The present analysis introduces a design procedure for vibration absorbers with more than a single dof and a single damper component. A requirement for the present procedure to work in the present case is that three real-valued roots for vanishing damper gain are reduced to two roots in the opposite gain limit, as a single root then approaches the imaginary axis along a reference quarter circular path. This design principle may possibly be extended to absorbers with more dofs, since the inverse point relation could as well be enforced for higher order characteristic equations.

\section{AcKnowledgements}

The comments and suggestions by the reviewers have substantially improved the article. Their contributions are hereby acknowledged.

\section{REFERENCES}

[1] N.W. Hagood and A. von Flotow, "Damping of structural vibrations with piezoelectric materials and passive electrical networks," Journal of Sound and Vibration, vol. 146, pp. 243-268, 1991. DOI: 10.1016/0022-460X(91)90762-9.

[2] A. Preumont, Vibration Control of Active Structures. An Introduction, Springer, Heidelberg, 2011. DOI: 10.1007/978-94-007-2033-6.

[3] S.O.R. Moheimani and A.J Fleming, Piezoelectric transducers for vibration control and damping, Springer, London, 2006. DOI: 10.1007/1-84628-332-9.

[4] O. Thomas, J. Ducarne and J-.F. Deü, "Performance of piezoelectric shunts for vibration reduction," Smart Materials and Structures, vol. 21, paper no. 015008 (16pp), 2012. DOI: 10.1088/0964-1726/21/1/015008. 
[5] K. Yamada, H. Matsuhisa, H. Utsuno and K. Sawada, "Optimum tuning of series and parallel LR circuits for passive vibration suppression using piezoelectric elements," Journal of Sound and Vibration, vol. 329, pp. 5036-5057, 2010. DOI: 10.1016/j.jsv.2010.06.021.

[6] M. Berardengo, A. Cigada, S. Manzoni and M. Vanali, "Vibration control by means of piezoelectric actuators shunted with LR impedances: Performance and robustness analysis," Shock and Vibration, vol. 2015, paper no. 704265 (30pp), 2015. DOI: 10.1155/2015/704265.

[7] P. Gardonio and D. Casagrande, "Shunted piezoelectric patch vibration absorber on twodimensional thin structures: Tuning considerations," Journal of Sound and Vibration, vol. 395, pp. 26-47, 2017. DOI: 10.1016/j.jsv.2017.02.019.

[8] N.E. duToit, B.L. Wardle and Kim SG, "Design considerations for MEMS-scale piezoelectric mechanical vibration energy harvesters," Integrated Ferroelectrics, vol. 71, pp. 121-160, 2005. DOI: $10.1080 / 10584580590964574$.

[9] N.E. duToit and B.L. Wardle, "Experimental verification of models for microfabricated piezoelectric vibration energy harvesters," AIAA Journal, vol. 45, pp. 1126-1137, 2007. DOI: $10.2514 / 1.25047$

[10] K. Nakano, S.J. Elliott and E. Rustighi, "A unified approach to optimal conditions of power harvesting using electromagnetic and piezoelectric transducers," Smart Materials and Structures, vol. 16, pp. 948-958, 2007. DOI: 10.1088/0964-1726/16/4/002.

[11] J.M. Renno, M.F. Daqaq and D.J. Inman, "On the optimal energy harvesting from a vibration source," Journal of Sound and Vibration, vol. 320, pp. 386-405, 2009. DOI: 10.1016/j.jsv.2008.07.029.

[12] J.P. Den Hartog, Mechanical Vibrations (4th edn). McGraw-Hill, New York (Reprinted by Dover, New York, 1985), 1956. ISBN 10: 0486647854.

[13] S. Krenk, "Frequency analysis of the tuned mass damper," Journal of Applied Mechanics, vol. 72, pp. 936-942, 2005. DOI: 10.1115/1.2062867.

[14] M. Zilletti, S.J. Elliott and E. Rustighi, "Optimisation of dynamic vibration absorbers to minimise kinetic energy and maximise internal power dissipation," Journal of Sound and Vibration, vol. 331, pp. 4093-4100, 2012. DOI: 10.1016/j.jsv.2012.04.023.

[15] S. Elias and V. Matsagar, "Research developments in vibration control of structures using passive tuned mass dampers," Annual Reviews in Control, vol. 44, pp. 129-156, 2017. DOI: 10.1016/j.arcontrol.2017.09.015.

[16] H. Garrido, O. Curadelli and D. Ambrosini, "Improvement of tuned mass damper by using rotational inertia through tuned viscous mass damper," Engineering Structures, vol. 56, pp. 2149-2153, 2013. DOI: 10.1016/j.engstruct.2013.08.044.

[17] Javidialesaadia A and Wierschem NE, "Optimal design of rotational inertial double tuned mass dampers under random excitation," Engineering Structures, vol. 165, pp. 412-421, 2018. DOI: 10.1016/j.engstruct.2018.03.033.

[18] Barredo E et al., "Closed-form solutions for the optimal design of inerter-based dynamic vibration absorbers," International Journal of Mechanical Sciences, vol. 144, pp. 41-53, 2018. DOI: $10.1016 /$ j.ijmecsci.2018.05.025. 
[19] L. Marian and A. Giaralis, "Optimal design of a novel tuned mass-damperinerter (TMDI) passive vibration control configuration for stochastically support-excited structural systems," Probabilistic Engineering Mechanics, vol. 38, pp. 156-164, 2014. DOI: 10.1016/j.probengmech.2014.03.007.

[20] T. Asai, Y. Araki and K. Ikago, "Structural control with tuned inertial mass electromagnetic transducers," Structural Control and Health Monitoring, vol. 25, paper no. e2059 (18pp), 2018. DOI: 10.1002/stc.2059.

[21] S. Krenk and J. Høgsberg, "Tuned resonant mass or inerter-based absorbers: unified calibration with quasi-dynamic flexibility and inertia correction," Proceedings of the Royal Society A - Mathematical, Physical and Engineering Sciences, vol. 472, paper no. 20150718 (23pp), 2016. DOI: $10.1098 /$ rspa.2015.0718.

[22] G.Y. Zhao, N. Alujevic, B. Depraetere and P. Sas, "Dynamic analysis and H-2 optimisation of a piezo-based tuned vibration absorber," Journal of Intelligent Material Systems and Structures, vol. 26, pp. 1995-2010, 2015. DOI: 10.1177/1045389X14546652.

[23] G.Y. Zhao, N. Alujevic, B. Depraetere, G. Piten and P. Sas, "Adaptive-passive control of structureborne noise of rotating machinery using a pair of shunted inertial actuators," Journal of Intelligent Material Systems and Structures, vol. 27, pp. 1584-1599, 2016. DOI: 10.1177/1045389X15600080.

[24] A. Gonzalez-Buelga, L.R. Clare, A. Cammarano, S.A. Neild, S.G. Burrow and D.J. Inman, "An optimised tuned mass damper/harvester device," Structural Control and Health Monitoring, vol. 21, pp. 11541169, 2014. DOI: 10.1002/stc.1639.

[25] H. Lu, T. Chai, C.G. Cooley, "Vibration properties of a rotating piezoelectric energy harvesting device that experiences gyroscopic effects," Journal of Sound and Vibration, vol. 416, pp. 258-278, 2018. DOI: 10.1016/j.jsv.2017.11.028.

[26] S.J. Elliott, M. Serrand and P. Gardonio, "Feedback stability limits for active isolation systems with reactive and inertial actuators," Journal of Vibration and Acoustics, vol. 123, pp. 250261, 2001. DOI: 10.1115/1.1350822.

[27] C. Collette and S. Chesné, "Robust hybrid mass damper," Journal of Sound and Vibration, vol. 375, pp. 19-27, 2016. DOI: 10.1016/j.jsv.2016.04.030.

[28] M. Zilletti, "Feedback control unit with an inerter proof-mass electrodynamic actuator," Journal of Sound and Vibration, vol. 369, pp. 16-28, 2016. DOI: 10.1016/j.jsv.2016.01.035.

[29] N. Alujevic, D. Cakmak, H. Wolf and M. Jokic, "Passive and active vibration isolation systems using inerter," Journal of Sound and Vibration, vol. 418, pp. 163-183, 2018. DOI: 10.1016/j.jsv.2017.12.031.

[30] Y.L. Cheung, W.O. Wong and L. Cheng, "Minimization of the mean square velocity response of dynamic structures using an active-passive dynamic vibration absorber," Journal of the Acoustical Society of America, vol. 132, pp. 197-207, 2012. DOI: 10.1121/1.4714362.

[31] S. Huyanan and N. Sims, "Vibration control strategies for proof-mass actuators," Journal of Vibration and Control, vol. 13, pp. 1785-1806, 2007. DOI: 10.1177/1077546307080031. 
[32] M. Berardengo, O. Thomas, C. Giraud-Audine, and S. Manzoni, "Improved resistive shunt by means of negative capacitance: new circuit, performances and multi-mode control," Smart Materials and Structures, vol. 25, paper no. 075033 (23pp), 2016. DOI: 10.1088/09641726/25/7/075033.

[33] M. Berardengo, O. Thomas, C. Giraud-Audine, and S. Manzoni, "Improved shunt damping with two negative capacitances: an efficient alternative to resonant shunt," Journal of Intelligent Material Systems and Structures, vol. 28, pp. 2222-2238, 2016. DOI: 10.1177/1045389X16667556.

[34] S. Krenk and J. Høgsberg, "Equal modal damping design for a family of resonant vibration control formats," Journal of Vibration and Control, vol. 19, pp. 1294-1315, 2013. DOI: 10.1177/1077546312446796.

[35] J. Høgsberg and S. Krenk, "Balanced calibration of resonant shunt cicuits for piezoelectric vibration control," Journal of Intelligent Material Systems and Structures, vol. 23, pp. 19371948, 2012. DOI: 10.1177/1045389X12455727.

[36] A.M. Gonzalez, A. Garcia, C. Benavente-Peces and L. Pardo, "Revisiting the characterization of the losses in piezoelectric materials from impedance spectroscopy at resonance," Materials, vol. 9, paper no. 72 (18pp), 2016. DOI: 10.3390/ma9020072.

[37] A. Morel, G. Pillonnet, Y. Wanderoild and A. Badel, "Dielectric losses considerations for piezoelectric energy harvesting," Journal of Low Power Electronics, vol. 14, pp. 244-254, 2018. DOI: $10.1166 /$ jolpe.2018.1562.

[38] P. Soltani, G. Kerschen, G. Tondreau and A Deraemaeker, "Piezoelectric vibration damping using resonant shunt circuits: an exact solution," Smart Materials and Structures, vol. 23, paper no. 125014 (11pp), 2014. DOI: 10.1088/0964-1726/23/12/125014. 